\title{
A Real-Time Rodent Tracking System for Both Light and Dark Cycle Behavior Analysis
}

\author{
Jane Brooks Zurn*+, Drew Hohmann+, Steven I. Dworkin^, Yuichi Motai* \\ *Department of ECE, University of Vermont, Burlington, USA \\ +MED Associates, Inc., Georgia, Vermont USA \\ ${ }^{\wedge}$ Department of Psychology, University of North Carolina, Wilmington, USA \\ \{jbzurn,ymotai\}@emba.uvm.edu
}

\begin{abstract}
Position tracking of rodents is useful and necessary to help elucidate the behavioral and physiological effects of genetic mutations, drug action, and environmental stimuli. In this paper we describe a real-time system developed to allow continuous overhead video monitoring of rodent behavior in a home cage environment, either in a daylight condition (light-cycle) using standard visible illumination or a night-time condition (dark-cycle), using overhead near infrared illumination (NIR). Due to the lack of research on the effects of NIR on rodent behavior, we also examined open-field locomotor activity under 880 $\mathrm{nm}$ and $940 \mathrm{~nm}$ wavelengths of NIR, as well as visible white light and a "dark" condition consisting of a very dim level of NIR. The experimental result validated the described system and robustly tracked the target rodent in the light cycle, and for high contrast conditions in the dark cycle.
\end{abstract}

\section{Introduction}

Activity monitoring is used to determine the behavioral effects of many experimental factors, including genetic mutations, drug influence, strain differences, brain lesions, disease, etc [1-5]. To determine the effects of these factors, it is necessary to identify and quantitatively record rodent behaviors that the researcher determines to be relevant. A nearly infinite number of behaviors may be of interest to the researcher, such as overall motion, rearing, grooming, sitting, sleeping, chewing, thigmotaxic travel, and so on [6]. Many measurement methods have been applied to this task, most commonly infrared beam crossing, activity wheel usage, and video tracking. Because video tracking has the advantage of being able to record the actual image of the rodent, it gives the flexibility of allowing both computer and manual classification of behavior patterns, and the ability to return to the data later and examine it for previously ignored behaviors. Examples of established systems developed for video monitoring of rodents are Activity Monitor 5 (MED Associates, Inc.), EthoVision ${ }^{\circledR}$ (Noldus Information Technology) [7], and the PhenoScan series (CleverSys, Inc.), and $[8,9]$. Other useful examples in the literature, not specifically for rodent tracking, are [10], which relies on shape differences for identifying object differences, and [11] which demonstrates a method for tracking multiple interacting objects.

Activity monitoring is utilized under either visible light or in the dark. Because research animals are housed in special facilities where environmental conditions (including light levels) can be controlled, convention has led to the identification of "day" and "night" to the "light cycle" and "dark cycle". This is necessary because rodent behavior is altered by light level [12]. Generally, rodents are most active during the dark cycle (for example, [12] and [13]), this is why many researchers choose to set the rodent's dark cycle to hours during daylight (a "reversed light - dark cycle").

Many types of tracking systems are capable of monitoring during the light cycle. However, tracking in the dark produces difficulties for typical video tracking systems, which are designed for use in visible light conditions [14]. A number of conditions necessitate monitoring of dark cycle behavior, including research on vision $[15,16]$ and the circadian cycle [12], therefore it is important to have methods of monitoring during the dark cycle. Because of the benefits of video tracking mentioned, we have developed a video tracking system for behavior monitoring that can operate during both the light and dark cycle, using near-infrared illumination (NIR) for the latter. The position of the rodent is determined using color thresholding combined with motion analysis. 


\section{Proposed System}

We propose the use of a CCD camera system as shown in Fig. 1. A IEEE-1394 interface connects the camera to a data-capturing computer, and an adjustable array of visible and infrared LEDs is fixed above the rodent chamber. This array can be switched between

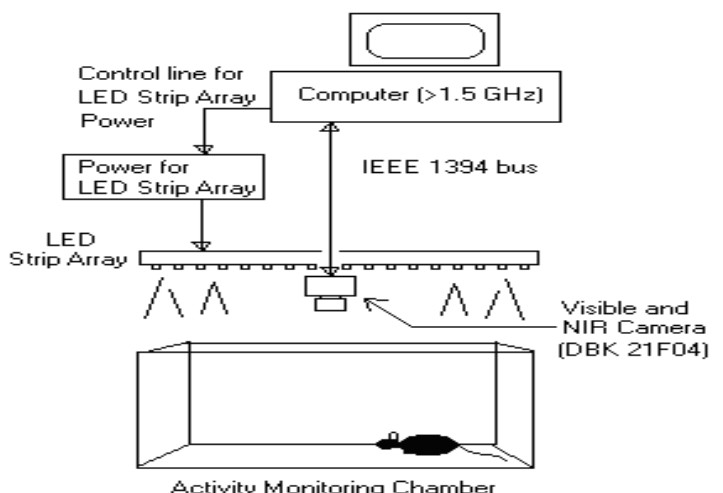

Fig. 1. Activity Monitoring System for Rodents

white light and NIR illumination for the light and dark cycles, respectively. A CCD camera is relatively inexpensive and the maximum wavelength response of the technology is about $300 \mathrm{~nm}$ (UV) to $1100 \mathrm{~nm}$ (near infrared) when no IR filter has been installed. This results in a potential illumination range of 750 $\mathrm{nm}$ to $1100 \mathrm{~nm}$. The camera used here is the DBK 21F04 from The Imaging Source (Bremen, Germany) and conforms to the DCAM standard, transmitting an image stream to the computer via an IEEE 1394a bus. The DBK 21F04 responds to a wavelength range from $<400 \mathrm{~nm}$ to $1000 \mathrm{~nm}$. A photo of the system is shown in Fig. 2.

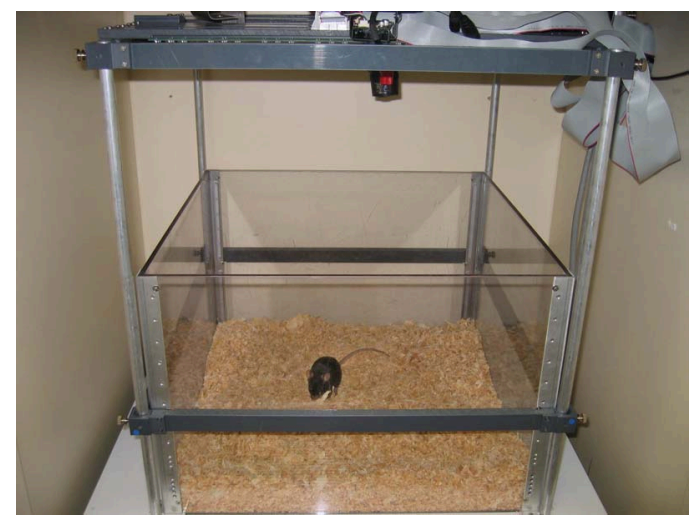

Fig. 2. Photo of Activity Monitoring System
Two commonly-available LEDs were tested for suitability $-880 \mathrm{~nm}$ and $940 \mathrm{~nm}$. It is interesting to note that some humans are capable of seeing into the NIR range (including the author). This serves to emphasize the importance of not overlooking the potential effects of NIR illumination on rodent behavior, since rodents' sensory capacities differ from humans.

\section{Testing Near Infrared Illumination Effects on Rodent Behavior}

Because video tracking requires a method of illumination, it is necessary to research the visual range of the rodents examined in order to ensure the illumination is outside that range. Rodents have been observed to have vision into the UV range (wavelengths $<390 \mathrm{~nm}$ ) [17], but it has generally been assumed that rodents cannot see in the NIR range ( $>$ $750 \mathrm{~nm}$ wavelength) due to the drop-off in visual sensitivity. However, some research has found retinal sensitivity and visual-evoked brain potentials to 936 $\mathrm{nm}$ light [18]. Therefore, if NIR illumination is to be used, it is necessary to examine its effects on behavior, if any. Since visible light suppresses locomotor activity when presented during the dark cycle ("night") in rodents $[8,9,10]$, our hypothesis is that activity under NIR illumination will be significantly greater than behavior under visible light.

To test the effects of NIR on rodent behavior, trials were run using 6 experimentally naïve SpragueDawley rats (Harlan International), each approximately 60-90 days old and housed in a reversed 12-hour light-dark cycle. Trials were run in the daytime, during the rats' dark cycle. Each trial consisted of 30 minutes spent in a standard open field activity monitoring chamber (MED Associates, Georgia VT USA). The activity was evaluated quantitatively for distance traveled, average velocity, and time spent resting.

\section{Tracking Algorithm}

The tracking algorithm utilizes color-threshold and image subtraction based algorithms. They are detailed as follows.

\subsection{Tracking During the Dark Cycle}

Several methods were examined for locating the mouse - color thresholding, image subtraction, and texture analysis. The algorithm used here relies most strongly on color thresholding. Color thresholding was very effective when the mouse coat color was of a 


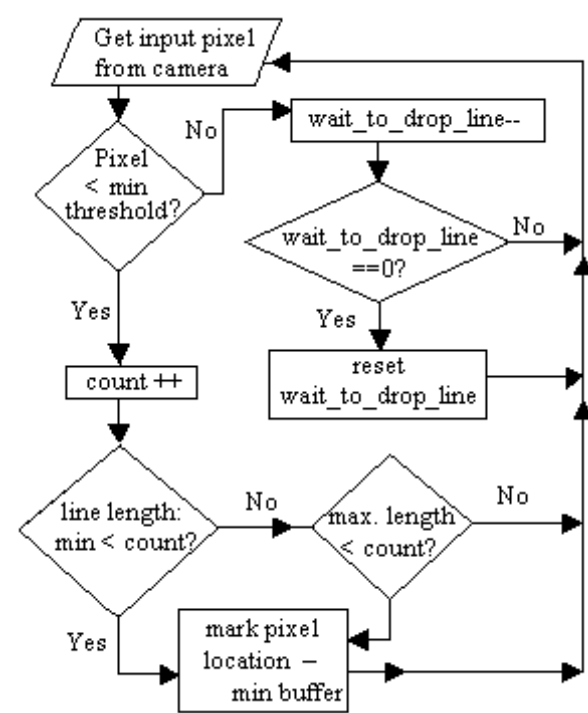

Fig. 3. Dark cycle algorithm for finding a rodent of high contrast to the background.

high contrast to the bedding (e. g. black coat on tan wood chip bedding). However, we found that for light of either $880 \mathrm{~nm}$ or $940 \mathrm{~nm}$ wavelengths, the infrared reflectance of a "white" mouse did not have a noticeably different color from the wood chip bedding. Therefore, a system using only color thresholding was limited to rodents with a coat color of high contrast to the background. This can be accomplished by using black rodents with woodchip bedding, or a solid gray colored base in the chamber. For image subtraction, a simple background snapshot was inadequate due to rodent tendency to dig and move bedding. A time-shifted image subtraction algorithm which subtracted the previously captured frame encountered the same difficulties as the color analysis because of the similarity in color between the white mouse and the bedding. Texture analysis initially held great promise, since the wood chip bedding had a great deal of texture variance and the white mice had very smooth coats. However, using a matrix-based edge extraction operation (such as a Laplacian function, as seen in [20]) slowed the computer processing to unacceptable levels. A fast processor ( $>$ than $2 \mathrm{GHz}$ ) would be necessary to implement such an algorithm.

The algorithm shown in Fig. 3 was used to locate the rodent. Because of the monochromatic light used for the NIR illumination, RGB information transmitted by the camera could be ignored and the intensity of each pixel could be used for the threshold. To reduce noise, the surrounding area of the pixel was also examined,

$$
\sum_{j=-2}^{j=2} P\left(x_{i+j,} y_{i+j}\right) / 5
$$

where $P$ is the intensity of the pixel (from $0=$ black to $255=$ white), and $x_{\mathrm{i}}$ and $y_{\mathrm{i}}$ are the pixel coordinates. If (1) results in a value less than the threshold, the pixel is marked and $i$ is incremented until all pixels in the frame are checked. The algorithm speed is greatly increased with little or no loss in accuracy when a subsampling of just every $5^{\text {th }}$ row is checked.

If the pixel was below the threshold for a black rodent (typically a value of 65 , where 255 was white and 0 is black), a count was started to determine the length of the line. The minimum limit was set to the minimum visible area of the rodent during rearing, the maximum length to $105 \%$ of the rodent's length from nose to rump when stretched out. If the line was within minimum and maximum limits, it was drawn on the screen, with the end result being a solid color blob designating the location of the mouse. A square marker was also drawn to indicate the location sent to the tracking program. Establishing minimum limits reduced noise, and maximum limits reduced the chance of the chamber sides being erroneously defined should they be exposed in the tracking region due to rodent burrowing and digging. The rodent's ears tended to be a different color than the threshold criteria, so we compensated for this by defining a distance that had to pass before a verified line was determined to end (the "wait_to_drop_line" variable in Fig. 3). This improved marking of the rodent's head. Overall, this resulted in marking of the rodent body with very little noise.

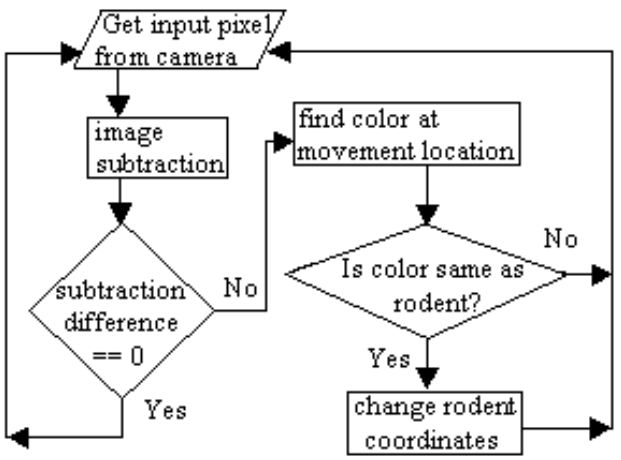

Fig. 4. Light cycle tracking algorithm.

\subsection{Tracking During the Light Cycle}

The light cycle algorithm was adapted from [19], where an average color vector was calculated for a region and compared to the color of interest. In [19] the algorithm analyzes the color of five regions of fixed relative position for face tracking; since many rodents are of a single color (with the notable exclusion of "hooded rats" which have a dark colored 
head and a light body), our algorithm only needs to assess one region. In the fixed conditions of laboratory rodent tracking, even hooded rats could be tracked by choosing one of the two body colors. The color of a region $R_{i}$ is determined using the following equation:

$$
P_{c}\left(r_{i}, q_{i}, b_{i}\right)=\sum_{(x, y)=R_{i}} \frac{P_{c}(r(x, y), g(x, y), b(x, y))}{\left|R_{i}\right|}
$$

where the color pixel $P_{c}$ has intensities of red $=r_{i}$, green $=g_{i}$, and blue $=b_{i}$ at location $(x, y)$ in region $R_{i}$. The size of the region was determined for the input requirements of Activity Monitor, for which the sampling distance of the number of rows and columns is based on the model of chamber that is being used.

The tracking algorithm for the light cycle uses a combination of image subtraction and color matching. The algorithm in Fig. 4 works for both light and dark rodent colors because they both have different spectral reflections from the background in the visible domain.

In this method, a region was first checked for motion. If motion was detected, the color vector was compared to the expected values using a goodness of fit ratio. Goodness of fit was determined as in [19] by calculating the ratio of each pixel color to its expected value:

$$
\psi_{i}=\frac{\max \left(P_{c}\left(\frac{r_{i}}{r i}, \frac{g_{i}}{g i}, \frac{b_{i}}{b i}\right)\right)}{\min \left(P_{c}\left(\frac{r_{i}}{r_{i}}, \frac{g_{i}}{\frac{g_{i}}{g i}}, \frac{b_{i}}{b i}\right)\right)}
$$

Where $\psi_{i}$ is the goodness of fit parameter, $r_{i}, g_{i}$, and $b_{i}$ are the measured color intensities and $\overline{r_{\mathbf{i}}}, \overline{g_{\mathbf{i}}}$, and $\overline{b_{i}}$ are the expected color intensities. If the goodness of fit was within acceptable limits, the location was marked. The new point was calculated using: $x_{i}=x_{i-1}$ $+\left(x_{i}-x_{i-1}\right) * 0.12$ and $x_{i}=x_{i-1}+\left(x_{i}-x_{i-1}\right) * 0.12$. This reduced "jumping" and smoothed the tracking of the rodent.

\section{Experiment and Evaluation Results}

\subsection{Effects of NIR Illumination on Rodents}

To determine the effects of NIR illumination on rodents, 6 white Sprague-Dawley rats (Harlan International) were tested under visible white light, $880 \mathrm{~nm}$ NIR illumination, $940 \mathrm{~nm}$ NIR illumination, and a dark condition consisting of no added light. These rats were also concurrently being used in a learning task. To test the results of the algorithms, the rodent behavior for the aforementioned lighting

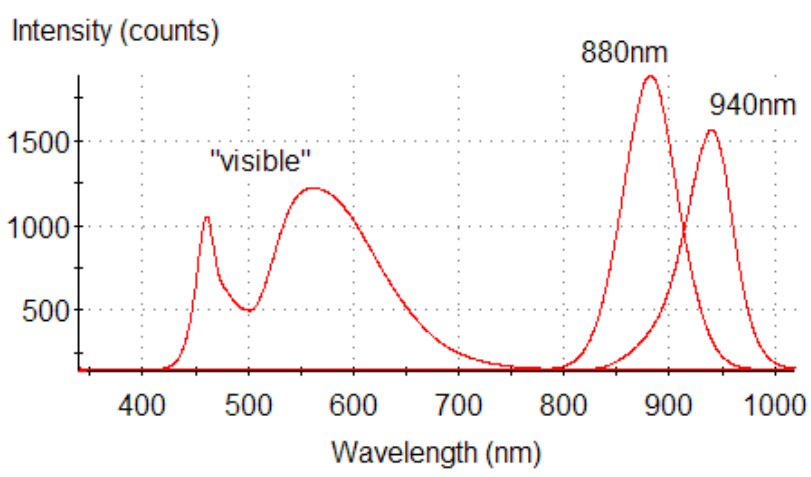

Fig. 5. Maximum spectral output of LED arrays at test height ( $\sim 50 \mathrm{~cm}$, depending on bedding distribution). The $\mathrm{x}$ axis represents light wavelength (350-1025 $\mathrm{nm})$, and the $y$ axis is a relative intensity measurement.

conditions was also run with horizontal IR crossbeams $(880 \mathrm{~nm}) 2 \mathrm{~cm}$ above the floor of the chamber to record movement. Since these crossbeams are widely used for tracking and had only 48 LEDs (the overhead illumination used 144 LEDs), it was assumed that the influence of the overhead NIR LEDs would be much greater than those of the crossbeams. The rats were maintained on a 12 hour reversed light/dark cycle (12 hours of light starting at $7 \mathrm{pm}$ ), and were tested during their dark cycle. The levels of light in the housing room were a maximum of 26 lux of red light during the dark cycle, and a maximum of 100 lux of white florescent light during the light cycle. The coordinates from the IR crossbeams and the video tracking were both transmitted to an existing rodent tracking analysis program (Activity Monitor 5, MED Associates, Inc.).

The emitted wavelengths of the illumination LEDs were measured using a photospectrometer (OceanOptics model USB2000, Dunedin, Florida). The spectral outputs of these are shown in Fig. 5, with the characteristics of the white LEDs. The peak measured wavelengths and standard deviations are in Table 1. The experimental procedure consisted of 30 minutes of placement in an open-field chamber under the light prescribed by the experimental condition. The floor of the chamber was white, and did not contain any bedding. The position of the rats was determined by the blocking of the horizontal NIR beams.

Visual qualitative observation of the trials revealed that the rodents in the white light condition generally suppressed locomotor activity after several minutes, while the other rats would ambulate for the entire time 


\begin{tabular}{|l|l|l|c|c|}
\hline $\begin{array}{l}\text { Table 1. Peak nominal and measured } \\
\text { wavelengths (nm) }\end{array}$ \\
\hline Nominal (nm) & $\begin{array}{l}\text { Low } \\
\text { white }\end{array}$ & $\begin{array}{l}\text { High } \\
\text { white }\end{array}$ & $\begin{array}{c}880 \\
\mathrm{~nm}\end{array}$ & $\begin{array}{c}940 \\
\mathrm{~nm}\end{array}$ \\
\hline Measured (nm) & 460.5 & 561.1 & 881.8 & 939.9 \\
\hline $\begin{array}{l}\text { Standard } \\
\text { Deviation (nm) }\end{array}$ & 276.4 & 355.8 & 430.1 & 338.6 \\
\hline
\end{tabular}

period. This suggests that "masking" [13] is present only for the white light. Thigmotaxis ("hugging the walls", a measure of rodent anxiety) was also elevated, but not significantly. The mean difference in rodent travel velocity between the NIR and white illumination conditions was not significant and the resting time was significantly larger for the white light (Tukey post-hoc test, $\mathrm{p}<0.027$ for each condition). Quantitative investigation of infrared illumination effects yielded a significant difference in rodent distance traveled between the visible white light and the dark and $940 \mathrm{~nm}$ infrared conditions (LSD posthoc test, $\mathrm{p}<.023$ and .033 , respectively), as in Table 2.

\begin{tabular}{|c|c|c|c|c|}
\hline \multicolumn{4}{|c|}{ Table 2. Mean ambulatory distance traveled } \\
\hline $\begin{array}{c}\text { Lighting } \\
\text { Condition }\end{array}$ & visible & $880 \mathrm{~nm}$ & $940 \mathrm{~nm}$ & dark \\
\hline $\begin{array}{c}\text { Mean } \\
\text { ambulatory } \\
\text { distance } \\
\text { (units) }\end{array}$ & 1021 & 1891 & 2067 & 1978 \\
\hline
\end{tabular}

It appears from the data that the difference between the means for $880 \mathrm{~nm}$ light and white light would reach significance with increased rodent sample size. From these data, it appeared advisable to use the 940 $\mathrm{nm}$ illumination for the dark cycle in order to increase the strength of the illumination difference between the light and dark cycles.

These data support the hypotheses that there is a significant difference between behavior in the dark and during the light, and that NIR illumination results in behavior that is not significantly different from dark cycle behavior.

\subsection{Results for Dark and Light Cycle Tracking}

A screen capture from the video program is shown in Fig. 6 for the NIR and white light illumination conditions, with a picture of the Activity Monitor screen for the same lighting condition using the output of the NIR crossbeams. We found that limiting the search area to $1-2 \mathrm{~cm}$ from the walls nearly eliminated mouse location error for the video tracking method; the thin line in (a) and (c) shows the boundary of the search area. The body of the mouse is located and filled in with color, and its location (shown with the small square) is transmitted to Activity Monitor. The video output of the camera was noticeably different for the visible illumination condition. However, this difference did not affect the tracking behavior. As can be seen from the figure, overall activity is greatly decreased under visible illumination. Although thigmotaxis appeared greater, it was not significant for the number of subjects (6) used in this study.

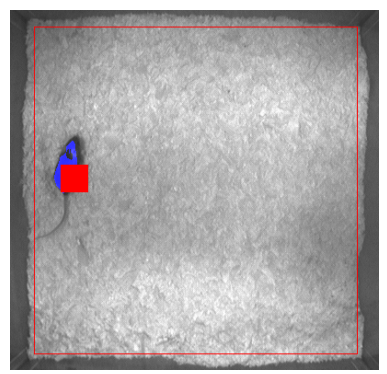

(a)

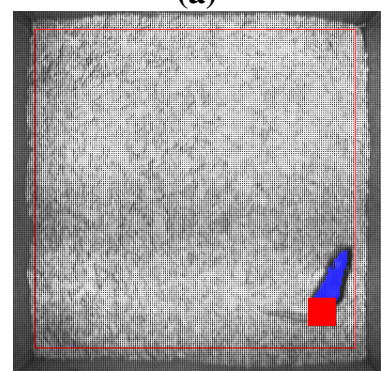

(c)

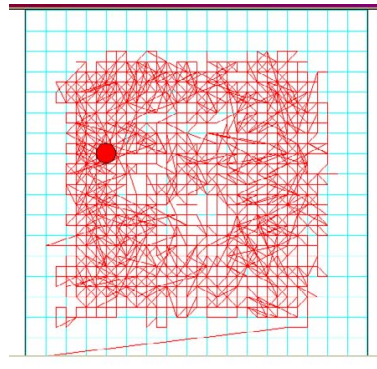

(b)

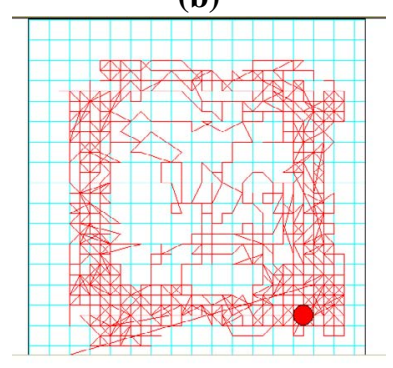

(c)
Fig. 6. Program and Activity Monitor output (a) and (b) $940 \mathrm{~nm}$ NIR illumination condition, (c) and (d) white illumination condition.

\section{Summary}

This system builds upon the work of [19] in that it adds an additional measure of position (motion detection) and extends the method to rodent dark cycle tracking, where such a method has not been used before. The motion detection improves the results for this application from those of [19] because it is still computationally simple, and it is capable of running faster by eliminating the need to perform calculations if the rodent is not moving.

The combination of color thresholding and motion analysis proved to be an economical, fast and effective method under daylight conditions for monitoring the location of rodents in an open field environment and a home cage environment. Thresholding was also very fast and effective under NIR illumination when the rodent color was of high contrast to the background.

NIR illumination was sufficient to track a rodent in a homecage when the rodent was of high contrast to 
the background color. However, rodent fur does not necessarily reflect NIR at the same rate as it reflects visible light. The white mice examined here (BALB/c, Charles River) reflected $880 \mathrm{~nm}$ and 940 $\mathrm{nm}$ NIR at essentially the same levels as the sawdust bedding (seen qualitatively from the camera, and measured using a photospectrometer), and so another means of distinguishing these mice from the background material appears to be necessary for future research.

\section{Acknowledgements}

Authors wish to thank MED Associates, Inc., Georgia, Vermont for interface software for the camera and Activity Monitor 5. Also we thank Shanna Babalonis, University of North Carolina, Wilmington, Department of Psychology, for testing and experimental design assistance.

\section{References}

[1] Y. A. Blednov, M. Stoffel, R. Cooper, D. Wallace, N. Mane, R. A. Harris, Hyperactivity and dopamine D-sub-1 receptor activation in mice lacking girk2 channels. Psychopharmacology. 159(4), pp. 370-378, 2002.

[2] M. A. Rigoulot, C. Leroy, E. Koning, A. Ferrandon, A. Nehlig, Prolonged low-dose caffeine exposure protects against hippocampal damage but not against the occurrence of epilepsy in the lithium-pilocarpine model in the rat. Epilepsia, 44(4). pp. 529-535, 2003.

[3] M. P. Paulus, S. C. Dulawa, R. J. Ralph, M. A. Geyer, Behavioral organization is independent of locomotor activity in 129 and C57 mouse strains. Brain Research, 835(1), pp. 27-36, 1999.

[4] J. J. Quinn, S. S. Oommen, G. E. Morrison, M. S. Fanselow, Post-training excitotoxic lesions of the dorsal hippocampus attenuate forward trace, backward trace, and delay fear conditioning in a temporally specific manner. Hippocampus, 12, pp. 495-504, 2002.

[5] G. Dell'Omo, E. Vannoni, A. L. Vyssotski, M. A. Di Bari, R. Nonno, U. Agrimi, H. P. Lipp, Early behavioural changes in mice infected with BSE and scrapie: automated home cage monitoring reveals prion strain differences. Eur. J. Neurosci., 16, pp. 735-742, 2002.

[6] C. Del Seppia, L. Mezzasalma, E Choleris, P. Luschi, S. Ghione, Effects of magnetic field exposure on open field behavior and nociceptive responses in mice. Behavioral Brain Research., 144, pp. 1-9, 2003.

[7] L. P. J. J. Noldus, A. J. Spink, and R. A. J. Tegelenbosch, "EthoVision: A versatile video tracking system for automation of behavioral experiments," Behavior Research Methods, Instruments, and Computers, vol. 33, pp. 398-414, 2001.
[8] K. Branson, V. Rabaud, and S. Belongie, "Three Brown Mice: See How They Run.," Visual Surveillance and Performance Evaluation of Tracking and Surveillance (VSPETS 03), 2003.

[9] C. J. Twining, C. J. Taylor, and P. Courtney, "Robust tracking and posture description for laboratory rodents using active shape models.," Behavior Research Methods, Instruments, and Computers, vol. 33, pp. 381-391, 2001.

[10] D. Magee and R. D. Boyle, "Detecting lameness in livestock using re-sampling condensation and multi-stream cyclic hidden Markov models.," Image and Vision Computing, vol. 20, pp. 581-594, 2002.

[11] Z. Khan, T. Balch, and F. Dellaert, "An MCMC-based Particle Filter for Tracking Multiple Interacting Targets," European Conference on Computer Vision (ECCV 04), 2004.

[12] A. Kramer, F.C. Yang, P. Snodgrass, X. Li, T. E. Scammell, F. C. Davis, C. J. Weitz, Regulation of Daily Locomotor Activity and Sleep by Hypothalamic EGF Receptor Signaling. Science, 294, pp. 2511-2515, 2001.

[13] N. Mrosovsky, P. Salmon, Learned Arbitrary Responses to Light in Mice Without Rods or Cones. Naturewissenschaften, 89, 525-527, 2002.

[14] Y. Motai and A. Kosaka, Concatenate Feature Extraction for Robust 3D Elliptic Object Localization. Proceedings of the ACM Symposium on Applied Computing, pp. 21-28, 2004.

[15] U. Redlin, N. Mrosovsky, Masking of locomotor activity in hamsters. J. Comp. Physio. A, 184, pp. 429-437, 1999.

[16] N. Mrosovsky, R. G. Foster, P. Salmon, Thresholds for masking responses to light in three strains of retinally degenerate mice. J. Comp. Physio. A, 184, pp. 423-428, 1999.

[17] G. H. Jacobs, J. A. Fenwick, G. A. Williams, Conebased Vision of Rats for Ultraviolet and Visible Light. Journal of Experimental Biology, 204, pp 2439-2446, 2001.

[18] M. T. Pardue, S. L. Ball, J. R. Hetling, V. Y. Chow, A. Y. Chow, N. S. Peachey, Visual Evoked Potentials to Infrared Stimulation in Normal Cats and Rats. Documenta Opthalmologica, 103, pp. 155-162, 2001.

[19] P. Fieguth and D. Terzopoulos, Color-Based Tracking of Heads and Other Mobile Objects at Video Frame Rates. Proceedings of the Conference on Computer Vision and Pattern Recognition, pp. 1063-69, 1997.

[20] H.R. Myler and A.R. Weeks, The Pocket Handbook of Image Processing Algorithms in C., p. 135. Prentice Hall, Upper Saddle River, New Jersey, 1993. 